\title{
CHBR: Contact History Based Routing in Time Varying Approach
}

Pravesh S Patel, Hemal Shah, Yogeshwar Kosta

U. V. Patel College of Engineering, Ganpat University,Mehsana Gujarat, India

pravesh.patel@ganpatuniversity.ac.in

U. V. Patel College of Engineering, Ganpat University,MehsanaGujarat, India

Hemal.shah@ganpatuniversity.ac.in

Marwadi Education Foundation, Rajkot, Gujarat, India

ypkosta@gmail.com

\begin{abstract}
In Delay tolerant network having intermittent connectivity so there is no guarantee of finding a complete communication path that connecting the source and destination. There no any end to end connectivity for delay-tolerant network selection of routing protocol is important to deliver the message in an efficient way and increases chance to deliver a message to the destination. Some existing routing protocols improve the delivery ratio but it also increases the overhead. Our paper proposed Contact History Based Routing (CHBR) that use Neighborhood Index and Time varying properties such as temporal distance, Temporal Diameter and centrality for benchmarking the existing routing protocol. First, temporal metrics are evaluated for synthetic and real trace data. Then CHBR protocol is compared with the Epidemic and PROPHET for delivery ratio, overhead and the number of messages dropped. This has been carried using Opportunistic Network Environment simulator under real and synthetic datasets.
\end{abstract}

\section{Indexing terms/Keywords}

DTN, Routing, CHBR, Neighborhood Index, PROPHET, Mobility model, Time Varying Graph, Time window size, RWP

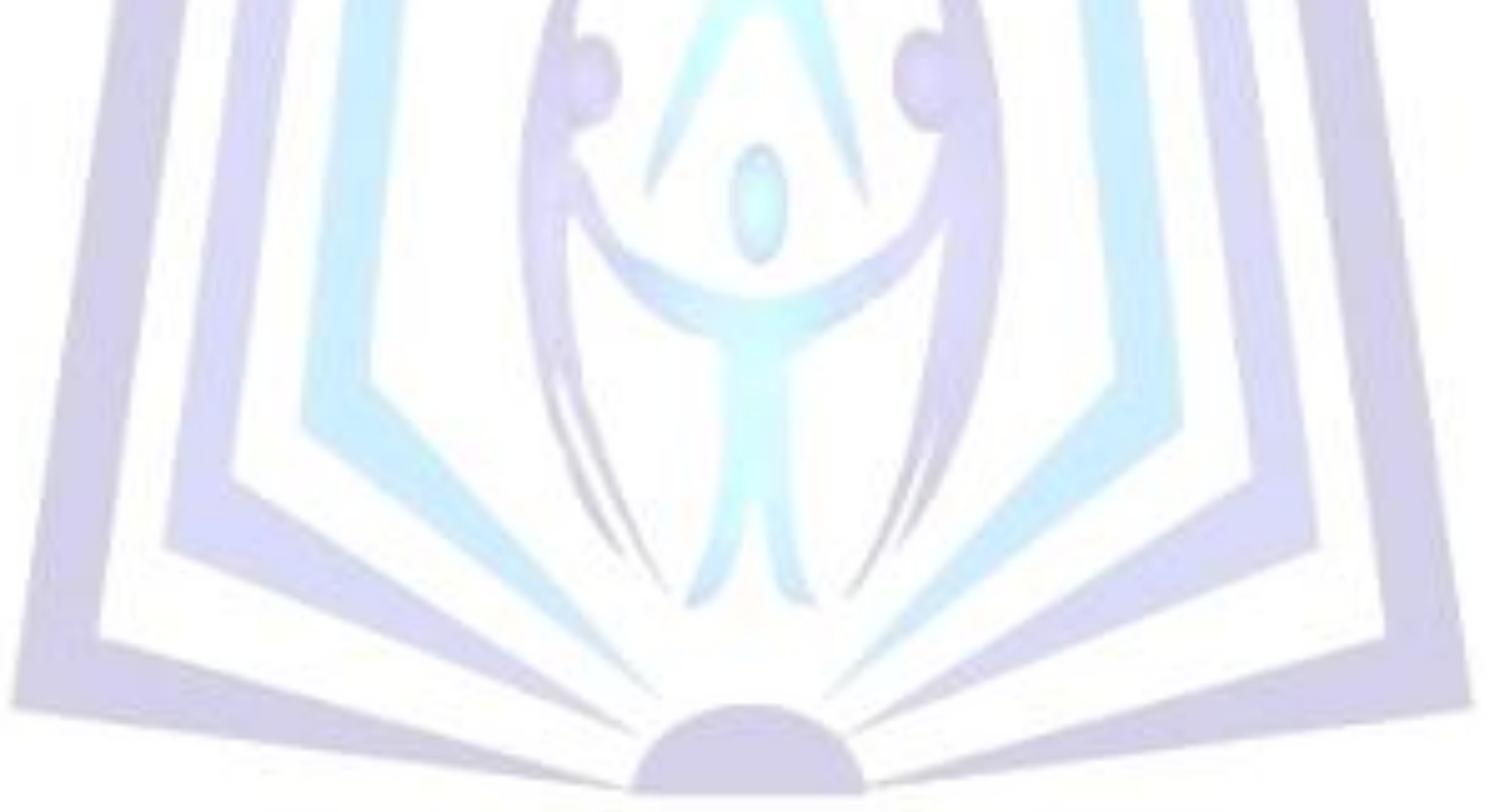

\section{Council for Innovative Research}

Peer Review Research Publishing System

Journal: INTERNATIONAL JOURNAL OF COMPUTERS \& TECHNOLOGY

Vol 13, No. 2

editor@cirworld.com

www.cirworld.com, www.ijctonline.com 


\section{Introduction}

Provide examples of relevant academic disciplines for this journal: E.g., History; Education; Sociology; Psychology; Cultural Studies;

Delay tolerant networks[1] or networks with intermittent connectivity networks are wireless networks often where, a communication path between a source node and destination node does not exist, either directly or through established routes by intermediate nodes. This situation occurs if the network is sparse and partitioned due to high mobility, or when the network extends over long distances; in these cases, the traditional routing protocols have been developed for ad hoc networks proved to be insufficient because they require the existence of end to end path in order to route the packets. Hence, researchers have proposed different techniques for exchanging the message in such environment based on store carry and forward mechanism.

Broad routing techniques in DTN environment can be classified as forwarding and replication. Forwarding technique only keeps the single copy of message in network. e.g. direct transmission, first contact [2]. This protocol utilizes the available resources optimally but suffers from lower delivery ratio. In replication, copies of each message spread across the network. E.g. epidemic routing[3]. This protocol give better delivery ratio[3] but, wastes resources such as bandwidth, buffer of network. Since, contacts are almost opportunistic, past history stored at each node may be use for future contact prediction. PROPHET[4] protocol uses the predictability values. These values are calculated using history of encounters and based on that forwarding decision is taken. With Prophet we get better delivery ratio compare to other routing but it increases overhead ratio. The work presented in this paper describes author's contribution as:

1. Temporal and Static representation of network

2. Proposed protocol based on contact history in time varying Approach

3. Performance analysis and comparison using ONE simulator

The rest of the paper is organized as section II introduces about routing protocols and mobility models, temporal graph simulator used respectively. Section III gives the information about the CHBR routing protocol and characteristics of it. Section IV discusses about the performance analysis our propose CHBR routing protocol with existing multi-copy routing scheme.

\section{Related Work}

Routing in delay-tolerant networking concerns itself with the ability to transport, or route, data from a source to a destination, which is a fundamental ability all communication networks must have. Delay- and disruption-tolerant networks (DTNs) are characterized by their lack of connectivity, resulting in a lack of instantaneous end-to-end paths. In these challenging environments, popular ad hoc routing protocols such as AODV[5] and DSR [6] fail to establish routes. This is due to these protocols trying to first establish a complete route and then, after the route has been established, forward the actual data. However, when instantaneous end-to-end paths are difficult or impossible to establish, routing protocols must take to a "store and forward" approach, where data is incrementally moved and stored throughout the network in hopes that it will eventually reach its destination[7][2]. A common technique used to maximize the probability of a message is successfully transferred is to replicate many copies of the message in hopes that one will succeed in reaching its destination[8].

DTN focus is mainly on being as efficient as possible with few available paths[4]. Hence routing protocols have been classified according to practical scenarios. These protocols can be classified according to methodology used to find destinations and whether replicas of messages are transmitted or not. Consequently routing protocols can be broadly characterized by

\section{Knowledge based (Forwarding) protocols \\ 2. Flooding based (Replica) protocols \\ 3. Coding based protocols}

The Direct Delivery Routing: is protocol that delivery message direct to destination, so until the destination is not in range of source, the source will carry the message. According to behavior of direct delivery routing the delivery ratio is to small compare to above discussed epidemic and prophet protocols

The epidemic routing protocol[9]: Messages propagate through the network like an outbreak of disease. This approach ensures that the message reaches its destination as much as possible, but it also wastes a lot of resources by unnecessary transfers of messages. 
The PROPHET [Probabilistic ROuting Protocol using History of Encounters and Transitivity] Routing[4] : is protocol is one of the routing algorithm that have been proposed to use their resource properly. Prophet introduced a metric called Delivery Predictability, $\mathrm{P}(\mathrm{a}, \mathrm{b})$ in $[0,1]$. This metric is calculated by each node a of the DTN network and for each known destination $b$ and will be used to decide which messages to be exchanged whenever two nodes meet.

The calculations of the delivery predictabilities have three parts. The first thing to do is to update the metric whenever a node is encountered, so that nodes that are often encounteredhave high delivery predictability. This calculation is shown in Eq. (i), where Pint in [0,1] is an initialization constant.

$$
P(a, b)=P(a, b) \text { old }+(1-P(a, b) \text { old }) \times \text { Pint }
$$

If a pair of nodes does not encounter each other in a while, they are less likely to be good forwarders of messages to each other, thus the delivery predictability values must age, being reduced in the process. The aging equation is shown in Eq. (ii), where $\alpha$ is the aging constant, and $k$ is the number of time units that have elapsed since the last time the metric was aged. The time unit used can differ, and should be defined based on the application and the expected delays in the targeted network.

$$
P(a, b)=P(a, b) \text { old } \times a k
$$

The delivery predictability also has a transitive property, that is based on the observation that if node $A$ frequently encounters node $B$, and node $B$ frequently encounters node $C$, then node $C$ probably is a good node to forward messages destined for node $A$ to. Eq. (iii) shows how this transitivity affects the delivery predictability, where $\beta \in[0,1]$ is a scaling constant that decides how large impact the transitivity should have on the delivery predictability.

\section{NECTAR protocol:}

$$
P(a, c)=P(a, b) \text { old }+(1-P(a, c) \text { old }) \times P(a, b) \times P(b, c) \times \beta \text { (iii) }
$$

NECTAR[10] protocol is based on the contacts history in order to create a Neighborhood Index and then determine the most appropriated route for DTNsIt uses the occurrence of an opportunistic contact to calculate a Neighborhood Index and spread messages in a controlled manner. During the contact period, nodes fist start the transmission of messages whose destination is the node that established the contact, then exchange information about the neighborhood (Neighborhood Index), and eventually forward other messagesThe NECTAR protocol can operate as the Epidemic Protocol. MinEpidemicLevel and MaxEpidemicLevel parameters were defined to allow this mode of operation and determine how many times a message can flood the network.

If the expression $(T T L)<M i n E p i d e m i c L e v e l$ is true, then the message will be transmitted to all neighbors. If MinEpidemicLevel parameter is set to one, only the source node will flood the network. If MinEpidemicLevel parameter is set to two, the source node will flood the network, as well as the source's direct neighbors. To avoid buffer overflow problem and network congestion, when the expression MinEpidemicLevel $<(T T L)<$ MaxEpidemicLevel is true, neighbors only accept this kind of message if the buffer occupation is below a certain threshold.

\section{Issues with NECTAR:}

With NECTAR the node having a higher Neighborhood index for destination is select for next rely node but, if there is no contact in future then ?NECTAR protocol is designed based on static graphs for Neighborhood index calculation thus; it reveals inaccurate information about node's neighbor. Hence, there is scope to extend the work of extracting Neighborhood index and propose a new routing protocol using Time Varying graph NECTAR is flooding based routing algorithm so each node require high buffer size, so there is scope for integration of good message drop policy

\section{DTN as Time Varying Graph}

Time varying graph (Temporal graph) can be represented in a sequence of time windows. Where for each of window we consider a snapshot of the network state at that particular time interval[11][12].It behaves like a Tool for understanding the dynamic properties of network over periods of time. Classical studies on social networks have focused on a static Representation of nodes and edges. After all static means, all edges are assumed to occur at the same time in temporal graph. However, in reality, such edges come and go across time.

Fig. (a)[13] shows the temporal graph of edge sequence. It consists six nodes that represented by $A, B, C, D, E$ and F. If we want to calculate the temporal distance then we have to follow the algorithm steps. Fig.(b) represents static representation of Fig.(a) 


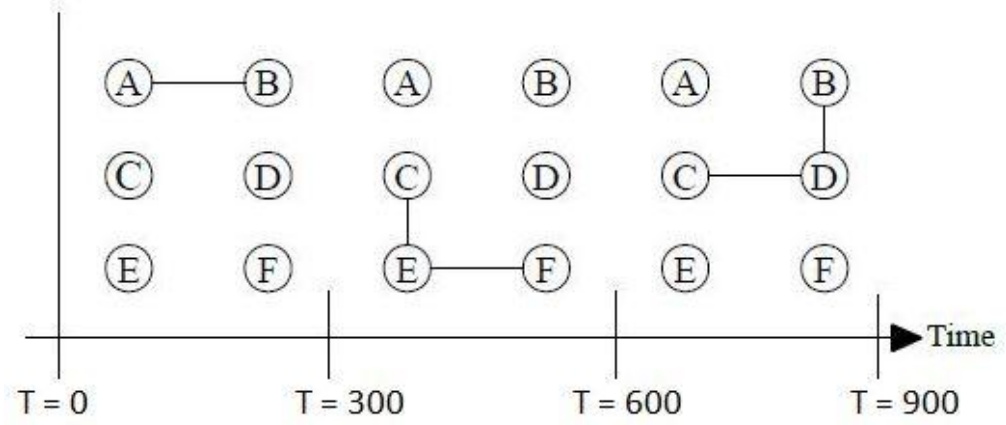

Fig.(a)Temporal Graph showing the sequence of edge connections

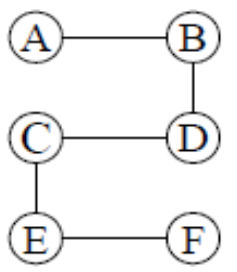

Fig. (b) Static graph constructed from Fig. (a)

\section{Temporal Metrics[14][15]:}

Temporal distance, temporal centrality and temporal diameter is important metrics that used to get dynamic properties of network

\section{CHBR routing}

Proposed protocol uses the occurrence of an opportunistic contact to calculate a Neighborhood Index and spread messages in a controlled manner. During the contact period, nodes first start the transmission of messages whose destination is the node that established the contact, then exchange information about the neighborhood (Neighborhood Index), and eventually forward other messages. The proposed routing protocol also uses the temporal distance and temporal diameter for efficient information diffusion.

The spread of the Neighborhood Index[10] allows a more accurate knowledge of network topology, which, inherently, aids the routing task. Although, to avoid unnecessary consumption of network resources, each node maintains a cache of neighbors it has contacted recently. Hence, nodes $i$ and $j$ can establish a new contact only after Tslot time slots (units of time) elapsed from the last contact between them, and if the Neighborhood Index table or message storage area (buffer) has changed

\section{Neighborhood Index calculation}

The Neighborhood Index is based on recent contact's history, in such a way that nodes that are frequent neighbors present a high Neighborhood Index.

Table 1: Neighborhood Index Parameters

\begin{tabular}{|c|l|}
\hline Parameter & \multicolumn{1}{|c|}{ Description } \\
\hline Contact $(i, j)$ & Define the amount of time slots that i and $\mathrm{j}$ remained in contact \\
\hline Hops $(i, j)$ & Express the number of hops required for $\mathrm{i}$ to reach $\mathrm{j}$ \\
\hline$T S$ & Current time Stamp \\
\hline ts_update $(i, j)$ & Time stamp of the last route update from $\mathrm{i}$ to $\mathrm{j}$ \\
\hline$\sigma$ & Define the aging constant \\
\hline$\omega$ & Weight applied to the known Neighborhood Index \\
\hline$N(i, j)$ & Neighborhood Index from i to destination $\mathrm{j}$ \\
\hline
\end{tabular}




\section{CHBR Algorithm based on Neighborhood index}

Input: Timewindow size, Weight function, aging constant

Output: Delivery ratio, Overhead Ratio, Message Drop Ratio

Definition: Overhead Ratio: (Relayed Message - Delivered Message) / Delivered Message

Algorithm Step:

1. Read the values of simulation time, time window size, aging constant and weight function.

2. If contact occurs ybetween $i$ and $j$ then

a) If contact counter is zero then set Neighborhood Index

$$
N(i, j) \text { is } 1 .
$$

b) Otherwise

Increment Neighborhood Index $N(i, j)$ by 1 .

3. Increment contact counter of node $i$ and node $j$

4. For each message of node $i$ do with each destination $d$

a) If $j$ is a not a destination ( $d$ )

$$
\text { If } N(j, d)>\mathrm{N}(\mathrm{i}, \mathrm{d}) \text { then }
$$

Compute distance matrix $=\operatorname{Hops}(j, d)+1$ and

Aging constant $(\sigma)=$ Amount of Time slot $j$ and $d$ are not in radio range

$$
N(i, d)=\frac{\operatorname{Contact}(j, d)}{(\text { Hops }(j, d)+1) \times\left(T S-t s_{-} \text {update }+1\right)^{\sigma}}
$$

Otherwise

$$
N(i, d)=\frac{N(i, d) \times \omega)+N(i, d)}{\omega+1}
$$

b) Otherwise, do not update Neighborhood index $N(i, d)$

5. Repeat step 4 for node $\mathrm{i}=\mathrm{j}$ and $\mathrm{j}=\mathrm{i}$

\section{Computation of Time Window size}

There are six nodes $(1,2,3,4,5,6)$ in network, if we wish to calculate Time window then find the total contact duration of each pair and also count the number of connections in each pair.

Table 2: Computation of Time Window size[13]

\begin{tabular}{|c|c|c|c|c|c|c|c|}
\hline $\begin{array}{c}\text { Node } \\
\text { ID }\end{array}$ & 1 & 2 & 3 & 4 & 5 & 6 & $\begin{array}{c}\text { Total contact time / } \\
\text { Total no of occurrences }\end{array}$ \\
\hline 1 & $0 / 0$ & $480 / 2$ & $720 / 3$ & $480 / 2$ & $960 / 4$ & $480 / 2$ & $3120 / 13$ \\
\hline 2 & $500 / 2$ & $0 / 0$ & $750 / 3$ & $250 / 1$ & $1000 / 4$ & $500 / 2$ & $3000 / 12$ \\
\hline 3 & $735 / 3$ & $490 / 2$ & $0 / 0$ & $490 / 2$ & $735 / 3$ & $245 / 1$ & $2695 / 11$ \\
\hline 4 & $235 / 1$ & $940 / 4$ & $1175 / 5$ & $0 / 0$ & $705 / 3$ & $470 / 2$ & $3525 / 15$ \\
\hline 5 & $1300 / 5$ & $260 / 1$ & $780 / 3$ & $520 / 2$ & $0 / 0$ & $1040 / 4$ & $3900 / 15$ \\
\hline 6 & $510 / 2$ & $510 / 2$ & $255 / 1$ & $1530 / 6$ & $1275 / 5$ & $0 / 0$ & $4080 / 16$ \\
\hline & & \multicolumn{7}{c}{$\sum T_{i j}\left(T_{\text {min }} T_{\text {max }}\right)$} & $20320 / 82$ \\
\hline
\end{tabular}




\section{Experimental Setup}

We used ONE simulator [18] to evaluate our proposed protocol CHBR and generate message state reports of for specific scenario of simulation Performance evolution of CHBR is compared with existing epidemic and Prophet routing.

Objective for simulation: to measure delivery ratio and overhead ratio of our proposed protocols and existing routing protocol. Delivery Ratio is define as ratio of total numbers of created message by the number of successfully delivered messages and Overhead Ratio is define by Equ (i)

$$
\text { Overhead Ratio }=\frac{\text { Relayed Messages }- \text { Delivered Messages }}{\text { Delivered Messages }}
$$

\section{Dataset Information}

Dataset being downloaded from Community Resource for Archiving Wireless Data at Dartmouth[19] (CRAWDAD) is a wireless network data resource for the research community site. Synthetic data is generated on ONE simulator and make compatible with existing real dataset.

Table 3: Dataset

\begin{tabular}{|c|c|c|}
\hline 1. & Name & RollerNet-INFOCOM09 \\
\hline & Location & During roller tour in Paris \\
\hline & Date & August 20, 2006 \\
\hline & Duration & 3096 seconds \\
\hline & Participants & 63 people participated to the rollerblading Tour \\
\hline & Address IDs & $\begin{array}{l}\text { ID 27-51 (25 iMotes)Staff members } \\
\text { ID 1-26 (26 iMotes)Skating associations } \\
\text { ID 52-62 (11 iMotes)A set of friends }\end{array}$ \\
\hline 2. & Name & Synthetic Data Set (Generate using ONE Simulator) \\
\hline $\bar{c}$ & Duration & 3096 Seconds \\
\hline & Participants & 63 Nodes \\
\hline & Mobility Model & Random Way Point (RWP) \\
\hline & Interface range & 100 meters \\
\hline & Address IDs & $0-62$ \\
\hline
\end{tabular}

First the temporal metrics is evaluated using Networkx tool for rollernet and synthetic (RWP) dataset . The evaluated temporal metrics of dataset is given in table 4

Table 4 : Evaluated Temporal Metrics

\begin{tabular}{|l|l|l|}
\hline Dataset Details & Rollernet & Synthetic_63 \\
\hline Tmin & 0 & 0 \\
\hline Tmax & 3096 & 3096 \\
\hline Total Nodes & 63 & 63 \\
\hline Total Number of Contacts & 2711107 & 576 \\
\hline Time Window Size & 15 & 71 \\
\hline
\end{tabular}




\begin{tabular}{|l|l|l|}
\hline Total timestamps & 207 & 44 \\
\hline Static distance & 1.22 & 3.64 \\
\hline temporal distance & 0.649 & 0.317 \\
\hline Diameter & 2 & 6 \\
\hline Degree centrality & $(51,0.1)$ & $(14,0.29)$ \\
\hline Betweenness centrality & $(51,0.004)$ & $(14,0.65)$ \\
\hline Clossness centrality & $(51,0.1)$ & $(15,0.43)$ \\
\hline
\end{tabular}

Simulation Settings of CHBR Routing for all dataset is given in table 5

Table 5 : Simulation Settings of all dataset

\begin{tabular}{|c|c|}
\hline Dataset & Rollernet/Synthetic \\
\hline Scenario.simulateConnections & False \\
\hline Scenario.updatelnterval & 3096 \\
\hline Scenario.endTime & 1 \\
\hline Scenario.nrofHostGroups & 10,10 \\
\hline Group.nodeLocation & StationaryMovement \\
\hline Group.movementModel & CHBR Router \\
\hline Group.router & 63 \\
\hline Group.nrofHosts & MessageEventGenerator \\
\hline Events.nrof & $1-2,3-4,5-6,7-8,9-10$ \\
\hline Events1.class & 0,62 \\
\hline Events1.interval & ExternalEventsQueue \\
\hline Events1.hosts & 15 / 71 \\
\hline Events2.class & \\
\hline Events2.filePath & \\
\hline Updatelnterval(window size) & roldat / Synthetic.dat \\
\hline
\end{tabular}


Performance evolution with rollernet and synthetic dataset

Delivery Ratio

\begin{tabular}{|c|c|c|c|}
\hline $\begin{array}{c}\text { Message } \\
\text { Event } \\
\text { Interval }\end{array}$ & $\begin{array}{c}\text { No of } \\
\text { Messages }\end{array}$ & Rollernet & Synthetic \\
\hline $1-2$ & 3096 & 0.65 & 0.07 \\
\hline $3-4$ & 1032 & 0.69 & 0.12 \\
\hline $5-6$ & 619 & 0.71 & 0.13 \\
\hline $7-8$ & 442 & 0.71 & 0.13 \\
\hline $9-10$ & 344 & 0.72 & 0.13 \\
\hline
\end{tabular}

In Synthetic dataset, number of connection edge is less due to randomize behavior of mobility model (RWP), also the contact period is very short, thus it impacts the delivery ratio compared to rollernet dataset with $\mathrm{CHBR}$ Routing.

Overhead Ratio:

\begin{tabular}{|c|c|c|c|}
\hline $\begin{array}{c}\text { Message } \\
\text { Event } \\
\text { Interval }\end{array}$ & $\begin{array}{c}\text { No of } \\
\text { Messages }\end{array}$ & Rollernet & Synthetic \\
\hline $1-2$ & 3096 & 45.39 & 100.81 \\
\hline $3-4$ & 1032 & 124.2 & 93.65 \\
\hline $5-6$ & 619 & 189.85 & 93.02 \\
\hline $7-8$ & 442 & 252.68 & 96.42 \\
\hline $9-10$ & 344 & 311.35 & 99.35 \\
\hline
\end{tabular}

In rollernet traces are for short duration and Neighborhood index is higher compared to RWP. Thus, CHBR drops more messages for rollernet. This leads to higher overhead compared to RWP.
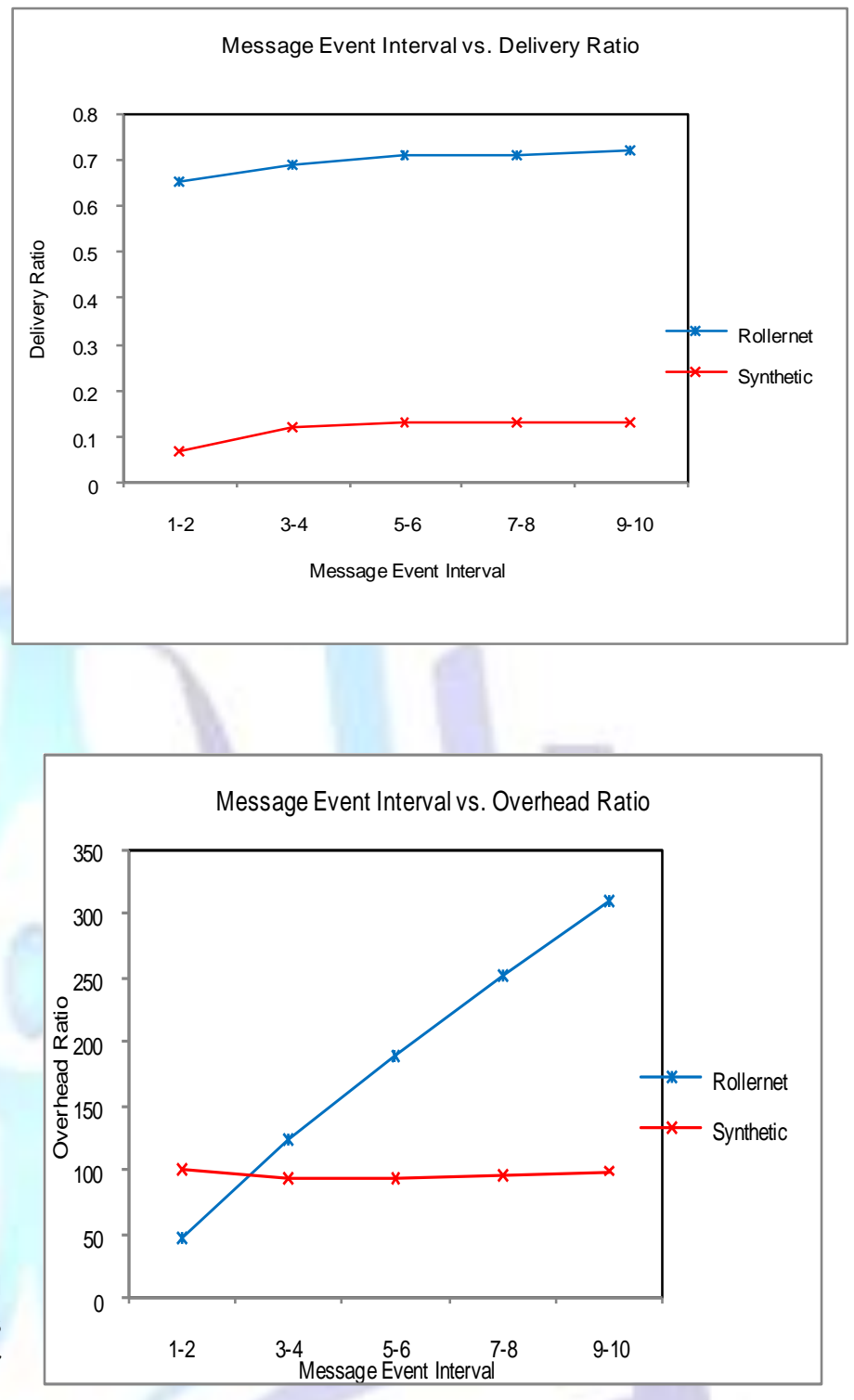

\section{Dropped Messages:}

\begin{tabular}{|c|c|c|c|}
\hline $\begin{array}{c}\text { Message } \\
\text { Event } \\
\text { Interval }\end{array}$ & $\begin{array}{c}\text { No of } \\
\text { Messages }\end{array}$ & Rollernet & Synthetic \\
\hline $1-2$ & 3096 & 89975 & 20383 \\
\hline $3-4$ & 1032 & 85051 & 8958 \\
\hline $5-6$ & 619 & 80476 & 4879 \\
\hline $7-8$ & 442 & 76204 & 3242 \\
\hline $9-10$ & 344 & 73799 & 2289 \\
\hline
\end{tabular}

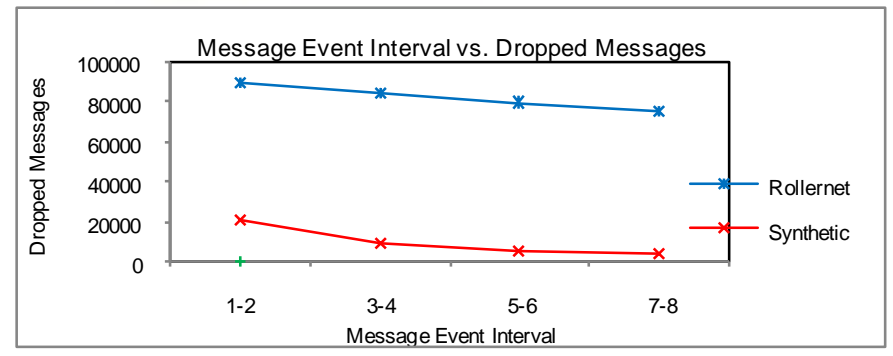

In Synthetic dataset, the number of connection edges is less due to randomize behavior of mobility model (RWP), also the contact period is very short. This leads to a lower node degree and in turn number of messages dropped for RWP compared to rollernet. 
Performance evolution of CHBR with other routing

Delivery Ratio:

\begin{tabular}{|c|c|c|c|c|}
\hline $\begin{array}{c}\text { Message } \\
\text { Event } \\
\text { Interval }\end{array}$ & $\begin{array}{c}\text { No of } \\
\text { Messages }\end{array}$ & Epidemic & CHBRouter & Prophet \\
\hline $1-2$ & 3096 & 0.41 & 0.65 & 0.40 \\
\hline $3-4$ & 1032 & 0.48 & 0.69 & 0.47 \\
\hline $5-6$ & 619 & 0.54 & 0.71 & 0.53 \\
\hline $7-8$ & 442 & 0.57 & 0.71 & 0.56 \\
\hline $9-10$ & 344 & 0.59 & 0.72 & 0.65 \\
\hline
\end{tabular}

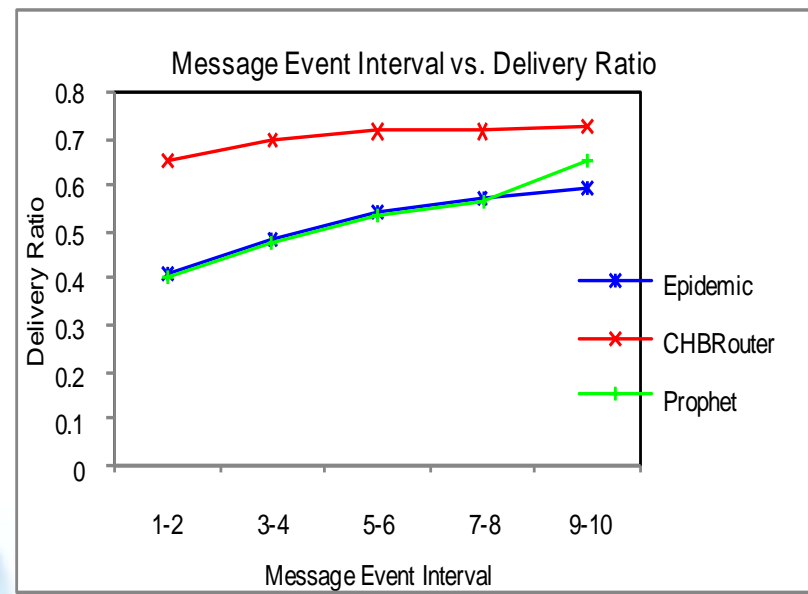

CHBR outperforms Epidemic and PROPHET due to the fact that the epidemic is a pure replication based technique. Thus, it drops the messages at relay nodes. PROPHET forwards the message copies based on probability and transitivity. It checks for contact probability values of all nodes in range and selects the node with high value. Thus, leads to poor delivery ratio compared to CHBR.

\section{Overhead Ratio:}

\begin{tabular}{|c|c|c|c|c|}
\hline $\begin{array}{c}\text { Message } \\
\text { Event } \\
\text { Interval }\end{array}$ & $\begin{array}{c}\text { No of } \\
\text { Messages }\end{array}$ & Epidemic & CHBRouter & Prophet \\
\hline $1-2$ & 3096 & 114.26 & 45.39 & 114.75 \\
\hline $3-4$ & 1032 & 276.34 & 124.2 & 281.74 \\
\hline $5-6$ & 619 & 392.9 & 189.85 & 396.37 \\
\hline $7-8$ & 442 & 489.88 & 252.68 & 496.29 \\
\hline $9-10$ & 344 & 586.13 & 311.35 & 532.76 \\
\hline
\end{tabular}

CHBR has lowest overhead due to selection of relay node based the higher neighborhood index. Thus, it spreads less number of copies into network compared Epidemic and PROPHET. This results into lower overhead ratio.

\section{Dropped Messages:}

\begin{tabular}{|c|c|c|c|c|}
\hline $\begin{array}{c}\text { Message } \\
\text { Event } \\
\text { Interval }\end{array}$ & $\begin{array}{c}\text { No of } \\
\text { Messages }\end{array}$ & Epidemic & CHBRouter & Prophet \\
\hline $1-2$ & 3096 & 144387 & 89975 & 141127 \\
\hline $3-4$ & 1032 & 135036 & 85051 & 133342 \\
\hline $5-6$ & 619 & 126915 & 80476 & 125768 \\
\hline $7-8$ & 442 & 119773 & 76204 & 120025 \\
\hline $9-10$ & 344 & 115864 & 73799 & 115686 \\
\hline
\end{tabular}

\section{Message Event Interval vs. Overhead Ratio}

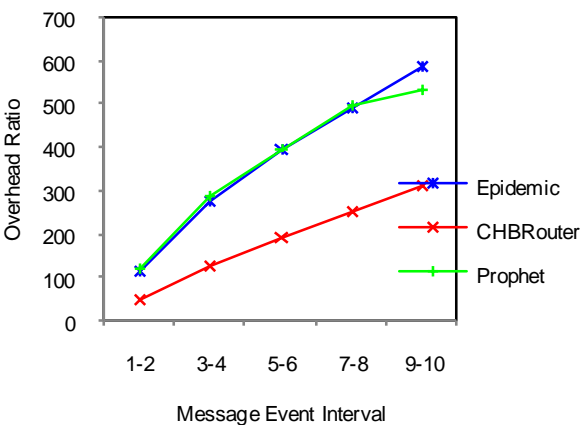

Message Event Interval

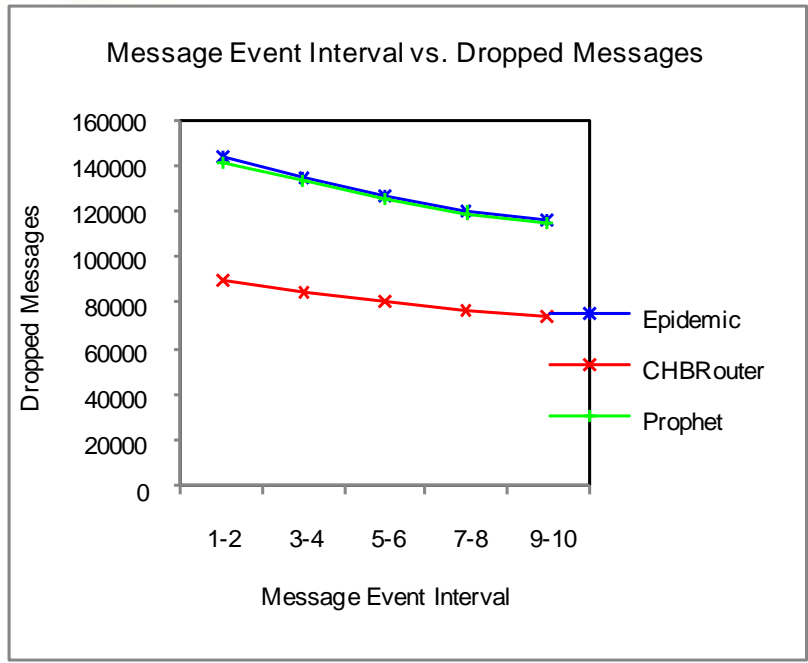

April 10,2014 
Epidemic is a pure replication based technique. It floods the network with message copies. Thus, it drops the messages at relay nodes. PROPHET forwards the message copies based on probability and transitivity. Though numbers of copies are flooded by PROPHET is more compared to CHBR, resulting in higher message drops.

\section{Conclusion}

CHBR uses a neighborhood index technique for forwarding the message copies. It does base on time intervals. This resulted into accurate information regarding per node neighbor's compared to similar probability based protocols. It has been further extended for suitable integration of drop policy-MOFO. It keeps track of forward count of messages per relay node. MOFO selects candidate for drop messages with highest forward count. Thus, it helps in reducing the overhead and improving the delivery ratio

\section{AKNOWLEGDEMENT}

We express our sincere gratitude to the management of Ganpat University - Mehsana for providing us research opportunities and their wholehearted support for such activities. Finally, our acknowledgement cannot end without thanking to the authors whose research papers helped us in making this research.

\section{REFERENCES}

[1] F. Warthman, Delay Tolerant Networks (DTN), prius.ist.osaka-u.ac.jp, no. March, 2003.

[2] T. Spyropoulos, K. Psounis, and C. S. Raghavendra, Efficient Routing in Intermittently Connected Mobile Networks: The Multiple-Copy Case, IEEE/ACM Transactions on Networking, vol. 16, no. 1, pp. 77-90, Feb. 2008.

[3] E. P. C. C. Jones, L. Li, P. A. S. S. Ward, and J. K. Schmidtke, Practical Routing in Delay-Tolerant Networks, in Proceeding of the 2005 ACM SIGCOMM workshop on Delay-tolerant networking - WDTN '05, 2005, vol. 6, no. 8, pp. 943-959.

[4] A. Lindgren, A. Doria, and O. Schelén, Probabilistic routing in intermittently connected networks, ACM SIGMOBILE Mobile Computing and Communications Review, vol. 7, no. 3, Jul. 2003.

[5] C. E. Perkins and E. M. Royer, Ad-hoc on-demand distance vector routing, in Mobile Computing Systems and Applications, 1999. Proceedings. WMCSA '99. Second IEEE Workshop on, 1999, pp. 90-100.

[6] D. B. Johnson and D. A. Maltz, Dynamic source routing in ad hoc wireless networks in Mobile Computing, 1996, pp. 153-181.

[7] J. Burgess, B. Gallagher, D. Jensen, and B. N. Levine, MaxProp: Routing for Vehicle-Based Disruption-Tolerant Networks, in Proceedings IEEE INFOCOM 2006. 25TH IEEE International Conference on Computer Communications, 2006, pp. 1-11.

[8] A. Vahdat and D. Becker, Epidemic Routing for Partially Connected Ad Hoc Networks. 2000.

[9] Amin Vahdat and David Becker, Epidemic Routing for Partially Connected Ad Hoc Networks, Tech. Rep. CS200006, 2000.

[10] E. C. R. de Oliveira and C. V. N. de Albuquerque, NECTAR: a DTN routing protocol based on neighborhood contact history in Proceedings of the 2009 ACM symposium on Applied Computing, 2009, pp. 40-46.

[11] J. Tang, M. Musolesi, C. Mascolo, V. Latora, and V. Nicosia, Analysing information flows and key mediators through temporal centrality metrics, in Proceedings of the 3rd Workshop on Social Network Systems, 2010, pp. 3:1-3:6.

[12] J. Tang, M. Musolesi, C. Mascolo, and V. Latora, Characterising temporal distance and reachability in mobile and online social networks, SIGCOMM Comput. Commun. Rev., vol. 40, no. 1, pp. 118-124, Jan. 2010.

[13] V. Patel and H. Shah, Characterization \& Evaluation of Temporal Metrics and Information Diffusion in Delay Tolerant Network, Ganpat University, 2012.

[14] J. Tang, M. Musolesi, C. Mascolo, and V. Latora, Characterising temporal distance and reachability in mobile and online social networks, ACM SIGCOMM Computer Communication Review, vol. 40, no. 1, Jan. 2010.

[15] A. Casteigts, P. Flocchini, W. Quattrociocchi, and N. Santoro, Time-Varying Graphs and Dynamic Networks, CoRR, Nov. 2010.

[16] J. Tang, M. Musolesi, C. Mascolo, and V. Latora, Temporal distance metrics for social network analysis, in Proceedings of the 2nd ACM workshop on Online social networks, 2009, pp. 31-36. 
[17] M. E. J. Newman, A measure of betweenness centrality based on random walks, Social Networks, vol. 27, no. 1, pp. 39-54, 2005.

[18] A. Keränen, T. Kärkkäinen, and J. Ott, Simulating Mobility and DTNs with the ONE (Invited Paper), Journal of Communications, vol. 5, no. 2, pp. 92-105, Feb. 2010.

[19] CRAWDAD: A community resource for archiving Wirelessdata at dartmouth. .

\section{Author' biography with Photo}

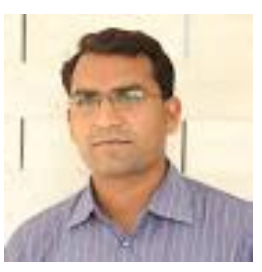

Pravesh Patel has done B.E Computer engineering in year 2006 and M. Tech Computer Engineering in year 2013 from Ganpat University. Currently he has working as assistant professor at U.V. Patel College of Engineering, Ganpat University, having 6 years of teaching experience.

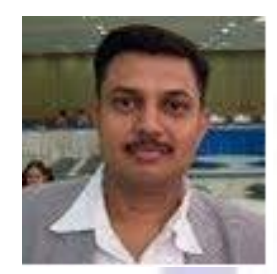

Hemal Shah is Ph.D. Scholar of Ganpat University, Gujarat India. He has done B.E. in year 1999 and M.E. Computer Engineering in year 2007 from DDIT Nadiad. Currently he has working as assistant professor at U.V. Patel College of Engineering, Ganpat University, having 12 years of teaching experience. He has carried out his PG- dissertation at Space application centre, Ahmadabad on TCP Performance Enhancing Proxies [PEP] for satellite links.

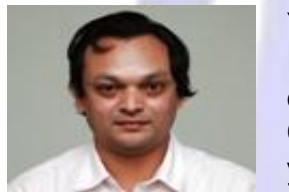

Yogeshwar Kosta is Professor and Director at Marwadi Education Foundation, Rajkot, Gujarat India. He has around 20 years of experience in Academic Teaching, Consultancy, Research and development in the field of Satellite Communications, RF and Microwave and Wireless Communications. He has worked as scientist at Space application centre, Ahmadabad around 8 years and Design and development of Ku-Band (Receiver) payload for the 